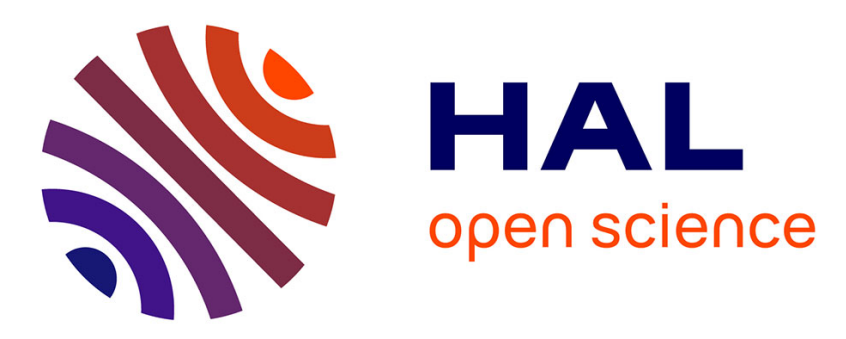

\title{
Fragilités et modes de régulation des figures de la compétence
}

Cathy Krohmer, Sophie Bretesché

\section{To cite this version:}

Cathy Krohmer, Sophie Bretesché. Fragilités et modes de régulation des figures de la compétence. Finance Contrôle Stratégie, 2013, 16 (2), pp.1-16. hal-01093191

\section{HAL Id: hal-01093191 https://hal.science/hal-01093191}

Submitted on 10 Dec 2014

HAL is a multi-disciplinary open access archive for the deposit and dissemination of scientific research documents, whether they are published or not. The documents may come from teaching and research institutions in France or abroad, or from public or private research centers.
L'archive ouverte pluridisciplinaire HAL, est destinée au dépôt et à la diffusion de documents scientifiques de niveau recherche, publiés ou non, émanant des établissements d'enseignement et de recherche français ou étrangers, des laboratoires publics ou privés. 
16-2 (2013)

Varia

Cathy Krohmer et Sophie Bretesché

\title{
Fragilités et modes de régulation des figures de la compétence
}

\author{
Avertissement \\ Le contenu de ce site relève de la législation française sur la propriété intellectuelle et est la propriété exclusive de \\ l'éditeur. \\ Les œuvres figurant sur ce site peuvent être consultées et reproduites sur un support papier ou numérique sous \\ réserve qu'elles soient strictement réservées à un usage soit personnel, soit scientifique ou pédagogique excluant \\ toute exploitation commerciale. La reproduction devra obligatoirement mentionner l'éditeur, le nom de la revue, \\ l'auteur et la référence du document. \\ Toute autre reproduction est interdite sauf accord préalable de l'éditeur, en dehors des cas prévus par la législation \\ en vigueur en France.
}

\section{revues.org}

Revues.org est un portail de revues en sciences humaines et sociales développé par le Cléo, Centre pour l'édition électronique ouverte (CNRS, EHESS, UP, UAPV).

\section{Référence électronique}

Cathy Krohmer et Sophie Bretesché, « Fragilités et modes de régulation des figures de la compétence », Finance Contrôle Stratégie [En ligne], 16-2 | 2013, mis en ligne le 12 juillet 2013, consulté le 10 décembre 2014. URL : http://fcs.revues.org/1362

Éditeur : Association FCS

http://fcs.revues.org

http://www.revues.org

Document accessible en ligne sur :

http://fcs.revues.org/1362

Document généré automatiquement le 10 décembre 2014

Tous droits réservés 


\section{Cathy Krohmer et Sophie Bretesché}

\section{Fragilités et modes de régulation des figures de la compétence}

Nous remercions les relecteurs anonymes pour leurs remarques constructives et leurs suggestions.

Nous remercions aussi les auteurs des huit monographies : Mathieu Detchessahar, Michel Devigne, Brigitte Charles-Pauvers, Bénédicte Geoffroy, Sarah Ghaffari, Bruno Henriet, Benoît Journé, Anne-France Kogan, Sophie Bretesché, Lionel Chambrier, Cathy Krohmer, Eric Melchior et Nathalie Schieb-Bienfait.

Depuis plus d'une vingtaine d'année, la compétence questionne les acteurs du monde de l'entreprise et de la recherche. Présentée par certains comme la révolution managériale offrant une alternative au modèle taylorien-fordien, elle suscite des débats passionnés au point de cristalliser de vives critiques ou inversement un inconditionnel engouement. Les outils de gestion des compétences ont été abondamment étudiés et aujourd'hui, l'analyse des pratiques constitue probablement une voie moyenne pour en appréhender les effets. C'est, comme nous y invite Segrestin (2004), le temps de l'épreuve des faits.

Au travers d'études empiriques, des chercheurs ont montré que le « modèle de la compétence » résiste difficilement. Le caractère multiforme des démarches (Defélix et al. 2007) est largement démontré au travers de typologies qui révèlent des configurations variées (Pichault et al. 2006) ou des catégories types (Parlier 2011). Les grilles de lecture retenues- le contextualisme pour l'étude de Pichault et al. (2006) - l'instrumentation de gestion, pour Parlier (2011) - permettent d'appréhender finement la diversité des pratiques. Toutefois, la dynamique autour de l'outil et plus précisément la façon dont les acteurs s'emparent de la démarche pour continuellement l'adapter, la contourner... en un mot la réguler, reste à préciser. L'étude présentée dans cet article s'inscrit dans cette perspective en précisant les fragilités de ces démarches et les modes de régulation alors développés (Reynaud 1997). Pour réaliser cette analyse, nous appréhendons ici les démarches compétences comme un cadre où se créent et se transforment des règles (Reynaud 2001). C'est donc bien ici la caractérisation des démarches et des dynamiques de l'action collective que chaque figure de la compétence initie qui nous intéresse.

Dans une première partie nous précisons le cadre d'analyse pour lire la diversité des figures des démarches compétences. Nous montrons ainsi que la variété des démarches repose non seulement sur des règles (au sens de Reynaud) dont les objets concernent le travail et/ou la gestion mais aussi sur des logiques différentes - logique d'adaptation des ressources humaines ou logique de développement des ressources humaines. Dans une deuxième partie, nous présentons nos choix méthodologiques, à savoir l'analyse qualitative de données secondaires issues de monographies relatant les démarches compétences de huit entreprises des Pays de la Loire. Dans une troisième partie, les quatre figures des démarches compétences sont spécifiées : la qualification locale, l'optimisation des ressources humaines, le déploiement du projet collectif et l'articulation locale/globale. Dans chaque figure apparaissent des fragilités et c'est au travers de régulations portées principalement par les salariés et le management de proximité que les démarches sont adaptées et négociées localement. Ces résultats sont discutés dans une quatrième partie.

\section{Un cadre d'analyse pour « lire » les démarches compétences}

Le caractère multiforme des démarches compétence est aujourd'hui largement avéré. Dès 1999, deux études l'attestent : l'une s'intéressant aux pratiques de Gestion Prévisionnelle des Emplois et des Compétences (GPEC) de 4 entreprises (Joyeau et Retour 1999), l'autre aux systèmes d'appréciation des compétences de 8 entreprises (Gilbert et Mader 1999). Par la suite, d'autres études confirment et prolongent ces résultats (Pichault et al. 2006 ; Parlier 
2011). Si chacun de ces travaux propose une distinction spécifique, il nous semble possible d'établir quelques consensus. Tout d'abord, nous distinguons les démarches compétence selon qu'elles visent à décrire le travail ou l'emploi (Parlier 2011 ; Pichault 2006). Ensuite, ces démarches sont soit dans une logique d'adaptation, soit de développement des ressources humaines (Joyeau et Retour 1999 ; Parlier 2011 et Pichault et al. 2006). Enfin, nous présentons les principales fragilités et régulations à l'occasion des démarches compétences.

\subsection{Les démarches compétence, de nouvelles règles de gestion et de travail}

Les démarches compétences fournissent aux acteurs un cadre d'action renouvelé (Dietrich 2002) en procédant à une redéfinition des règles de l'action collective (Reynaud 2001). Ces règles, dont les objets sont multiples (Reynaud 1988), concernent les méthodes de travail qui précisent les moyens pour aboutir au produit recherché, les critères qualité acceptés ou encore les précautions à prendre... Ces règles fixent aussi les conditions d'accès aux postes, les promotions, le niveau des salaires... On retrouve ici la diversité des objets de la démarche compétence. Celle-ci est effectivement au cœur des politiques de gestion des ressources humaines et de l'organisation du travail. Pour rendre compte de ce double aspect, une distinction est souvent faite entre gestion des compétences, qui serait une technique de gestion des ressources humaines, et gestion par les compétences, qui reflèterait un mode de management (Parlier 2011). Ces démarches englobent alors la dimension emploi et la dimension travail-activité (Autès 2004), marquent un nouveau rapport salarial et une nouvelle approche de l'activité (Segrestin 2004) ou encore portent en elles une double logique gestionnaire et productive (Oiry et Sulzer 2002). C'est bien sur ces deux dimensions que portent les règles produites à l'occasion des démarches compétences. Pour appréhender ces deux dimensions, nous proposons ici de parler de règle de gestion et de règle de travail. Les règles de gestion renvoient à l'ensemble des règles qui constitue la relation d'emploi et les règles de travail à celles relatives à l'activité productive. Les démarches compétences combinent plus ou moins ces deux règles. Dans la pratique, une démarche repose plutôt sur une règle de gestion ou une règle de travail sans toutefois exclure totalement l'autre règle.

Précisons à présent ce que recouvrent plus spécifiquement ces règles. Après un certain nombre d'expérimentations, les règles de gestion sont actuellement inscrites durablement dans les pratiques des entreprises (Gilbert 2003) et s'accompagnent d'une instrumentation conséquente (Defélix et al. 2007 ; Detchessahar et Journé 2007). Ces pratiques recouvrent l'évaluation, la formation, la gestion de carrières ou encore l'identification des compétences matérialisée par un outil particulièrement emblématique : le référentiel de compétences (Jouvenot et Parlier 2005 ; Retour et Rapiau 2006).

Envisagée comme une règle de travail, la compétence est perçue comme l'élément déterminant face aux nouveaux enjeux des entreprises (Bouteiller 1997). Zarifian (2001) repère trois mutations dans le contenu même du travail : l'évènement, la communication et le service. La compétence se traduit au travers d'une autonomie des salariés qui se mobilisent dans les situations (Paradeise et Lichtenberger 2001). Cette mobilisation engage alors leur responsabilisation (Zarifian 1988 ; Richebé 2002) qui suppose une disponibilité mentale, une implication dans le travail, un engagement psychique fort tendu vers une volonté de bien faire et de se sentir responsable de ses actes (Everaere 2000). La démarche insère aussi le salarié dans un ensemble d'interdépendances (Paradeise et Lichtenberger 2001). La coopération, la communication dans le travail devenu souvent collectif, se révèlent alors être des exigences de premier plan.

\subsection{Logique d'adaptation et logique de développement}

Une seconde distinction peut être opérée selon la logique de la démarche (Joyeau et Retour 1999 ; Pichault et al. 2006 ; Parlier 2011). Pour Joyeau et Retour (1999), la démarche peut avoir une visée de contrôle se traduisant par une adaptation des ressources aux besoins de l'entreprise ou une visée d'autonomie où les ressources humaines sont envisagées comme un facteur de développement économique. Parlier (2011) distingue les démarches unilatéralement 
profitables de celles mutuellement profitables. Dans le premier cas, il s'agit pour l'entreprise de renforcer ses exigences auprès de ses salariés. Dans le second cas, au contraire elle permet d'instaurer un nouveau compromis social où l'engagement des salariés est reconnu.

Dans une logique d'adaptation des ressources humaines, la démarche compétence est initiée afin d'ajuster en continu l'organisation du travail ou les compétences individuelles aux exigences du marché. Dans le cas d'organisations flexibles, c'est un contexte d'adaptation à la demande client qui détermine la mise en œuvre des démarches compétences. Les compétences « requises » (Retour 2005) sont définies au regard des exigences du marché et des critères qu'il affecte au produit en termes de coûts, de qualité et de délai. Cette mise en relation quasi directe entre l'organisation de l'activité et la demande du marché met à mal des formes tayloriennes du travail caractérisées par l'absence de déviation des producteurs par rapport aux schémas conçus à leur amont ou de retour de l'usager ou du client sur les modes de fabrication. En définitive, il revient au salarié la responsabilité de la gestion des exigences de production. Comme le souligne Zarifian (2009), la « mise en rythme du travail exercé par les commandes venant du marché » requiert des modes de coopération fondées sur des valeurs communes associées aux objectifs du travail et c'est d'ailleurs dans ce sens que les démarches compétences s'inscrivent comme de nouvelles règles sur lesquelles se constituent les accords au sein des équipes de travail.

Dans une logique de développement, c'est le «projet » qui active un cycle de développement dans lequel les compétences individuelles détenues ou mobilisées (Retour 2005) constituent un maillon clé. La construction d'un projet collectif pour l'entreprise initie une forme de contrat tacite entre le développement de l'entreprise et l'engagement de l'individu dans ce cadre d'action. La rhétorique du «parcours professionnel » et de la « gestion des compétences », au sens de «portefeuille », signifie le régime d'engagement requis, à savoir l'acquisition de nouvelles compétences censées assurer la compétitivité de l'entreprise et l'employabilité des salariés. Le temps de la formation continue et le temps d'apprentissage s'inscrivent dans une logique de "mobilisation subjective » (Monchatre 2004) qui redéfinit les conditions de l'intégration sociale et pose de nouveaux principes en matière de gestion de carrières. L'individu est par conséquent incité à entreprendre un cheminement professionnel balisé par les perspectives stratégiques de l'entreprise. Par homologie, trajectoire d'entreprise et parcours individuel sont appréhendés de façon itérative, dans une logique «gagnant-gagnant ». La projection dans l'avenir et la capacité à faire valoir et reconnaître ses compétences fondent en quelque sorte la nouvelle responsabilité qui incombe à l'individu dans l'entreprise. La démarche compétence est alors censée promouvoir un processus synchrone entre le développement de l'entreprise et l'acquisition de compétences spécifiques par les salariés, c'est d'ailleurs dans ce sens que le MEDEF promeut un nouvel échange salarial fondé sur la compétitivité de la firme et l'employabilité des salariés (Reynaud 2001).

Logique d'adaptation et logique de développement requièrent des types de mobilisation des salariés différents. Tantôt les démarches compétences ont pour objectif de valider la capacité des salariés à faire face en temps réel aux exigences du système productif, tantôt elles s'articulent étroitement avec des cycles de développement économiques planifiés sur le moyen terme et dans cette perspective, elles visent la «mise en mouvement» des salariés.

\subsection{Des démarches fragiles, objet de régulation}

Dans ce nouveau système de règle, le regard porté sur la main-d'œuvre est renouvelé. Souvent comparée à la qualification, la compétence se révèle beaucoup plus fragile. C'est ainsi que les compétences liées au résultat sont désormais mortelles (Reynaud 2001). Les compétences deviennent aussi difficilement transférables (Reynaud, 2001) d'une entreprise à une autre. De caractère contingent et local (Courpasson et Livian 1991), « rien ne permet de dire que les ouvriers sont propriétaires des compétences qui leur sont reconnues» (Monchatre 2004). Enfin, une autre critique adressée à l'encontre du modèle compétence est l'accent mis sur la personne dans le couple «homme-situation de travail » (Gilbert 1994) traduisant une individualisation de la relation d'emploi. Pour Courpasson et Livian (1991), le salarié est désormais seul face à l'entreprise. 
Parmi ces principales fragilités de la compétence identifiées dans la littérature, certaines sont atténuées par des régulations. C'est ainsi que le management de proximité invente de nouvelles règles pour désamorcer les tensions qui résultent de la démarche compétence (Monchatre 2002). Plus particulièrement, le management intervient au niveau de l'articulation entre l'exigence d'individualisation contenue dans la démarche et la nécessité d'une dimension collective dans le travail. Par exemple, Richebé (2002), qui étudie la mise en place d'un nouveau dispositif d'évaluation et de rémunération des compétences, montre que les détournements réalisés par le management de proximité des outils d'évaluation des compétences «permettent de préserver - au moins en partie - ces logiques collectives ». Les salariés résistent aussi à la démarche. Monchatre (2004), dans le cadre d'une étude portant sur l'application de l'accord ACAP 2000 dans la sidérurgie, identifie trois stratégies individuelles d'adaptation : le refus - les ouvriers ne se soumettent pas à la nouvelle discipline ; l'installation - les ouvriers sont à la recherche de solutions qui permettent de cumuler les satisfactions trouvées dans l'institution en dépit des contraintes imposées ; et enfin, l'adoption - ici les ouvriers s'efforcent de jouer le rôle du parfait ouvrier.

Enfin, les responsables des ressources humaines peuvent aussi transformer les règles en présence (Havard et Krohmer 2008). Eustache (2001) montre ainsi que dans le cadre de l'application d'une grille de classification et de rémunération en fonction des compétences, un dialogue s'instaure entre les managers de proximité et la fonction ressources humaines qui aboutit à l'établissement d'une nouvelle règle salariale.

Les fragilités et ses régulations ici relevées sont décrites soit en référence au modèle de la compétence (par exemple l'article de Reynaud, 2001), soit à partir d'exemples précis de pratiques d'entreprises (Richebé 2002 ; Monchatre 2002 et 2004 ; Eustache 2001). Or, comme nous l'avons vu, les démarches sont largement multiformes et donc susceptibles d'être porteuses de fragilités et de régulations spécifiques.

\section{Une démarche de recherche fondée sur l'analyse des pratiques}

Pour comprendre en profondeur les éléments distinctifs des démarches compétences, nous avons conduit une recherche qualitative reposant sur l'analyse de plusieurs monographies. Les diverses études de cas ont été réalisées et rédigées sous forme de monographies par des chercheurs dans le cadre du projet RECOR (Ressources COmpétence en REgion) financé par la DRTEFP, la DREAL des Pays de la Loire et le FSE et porté par l'Ecole des Mines de Nantes. Chaque équipe de chercheurs a rédigé une monographie d'environ une vingtaine de pages reprenant le contexte d'émergence de la démarche, une description de la démarche et de l'outil gestionnaire et une présentation des principaux effets de la démarche sur l'organisation et les différents acteurs. La phase d'enquête s'est étalée de 2006 à 2008. Au final des documents internes comme les descriptifs des emplois-types, les référentiels de compétences, les grilles d'entretiens etc. ont été collectées et plus de 260 entretiens semi-directifs ont été réalisés. Les interviews ont été menés auprès des dirigeants (5\% des entretiens), des personnes en charge des ressources humaines ( $6 \%$ des entretiens), du ou des consultants ( $4 \%$ des entretiens) ayant participé à l'élaboration et/ou l'amélioration de la démarche compétence, la hiérarchie intermédiaire ( $25 \%$ des entretiens) et les salariés (60\% des entretiens). Les équipes ont restitué les résultats auprès des acteurs de l'entreprise.

Sur les 13 cas produits dans le cadre du projet RECOR, nous avons sélectionné les 8 monographies suivantes : Construction (Charles-Pauvers et Schieb-Bienfait 2008), Fenêtre (Henriet et Krohmer 2008), Logemer (Bretesché et Chambrier 2007), Matériel (CharlesPauvers et Schieb-Bienfait 2008), Micro-Contrôle (Devigne, Detchessahar et Geoffroy 2007), Modeluxe (Ghaffari et Kogan 2007), Silicone (Journé et Melchior 2008) et Toutes-Graines (Bretesché et Devigne 2009). Les monographies sélectionnées portent sur des entreprises dont le secteur d'activité est varié (industrie automobile, textile, construction, industrie agroalimentaire....) qui disposent d'un référentiel de compétence et dans lesquelles au moins deux campagnes d'évaluation des salariés ont été réalisées (cf. encadré 1). 
Construction (Charles-Pauvers et Schieb-Bienfait 2008) est une entreprise d'une centaine de salariés spécialisée dans la construction de maisons individuelles et de bâtiments agricoles. Depuis 20 ans, l'entreprise a connu une croissance régulière et continue (chiffres d'affaires en 2006 : 7 millions d'euros). L'entreprise s'est diversifiée sur de nouveaux domaines d'activité stratégique, notamment le bois. En 2000, le directeur s'engage dans une démarche compétence nommée « démarche d'entreprise apprenante ».

Fenêtre (Henriet et Krohmer 2007) fabrique des huisseries en PVC, aluminium et bois pour une clientèle de professionnels. Cette entreprise familiale de 300 salariés est passée d'une production artisanale à une production industrielle et a connu sur les dix dernières années une progression très rapide. Cette évolution s'est accompagnée d'une réflexion sur un process industriel plus flexible et plus fiable et d'une mise à plat du système de gestion des ressources humaines qui s'est traduite par l'élaboration d'une démarche compétence en 2001.

Logemer (Bretesché et Chambrier 2007) est un organisme de logement social de 150 salariés qui, à l'image des organisations du secteur de l'habitat social, connaît depuis une vingtaine d'années de profondes mutations. Le service à l'habitant constitue désormais un axe d'évolution de l'action des bailleurs qui interroge les modes de gestion, l'organisation des métiers et les cultures professionnelles. En 2002, le directeur développe une approche par les compétences afin de favoriser de nouvelles pratiques fondées sur la qualité de service.

Depuis cinq ans, la direction de Matériel (Charles-Pauvers et Schieb-Bienfait 2008) a choisi de diversifier les activités de l'entreprise passant d'un métier unique de la location de gros matériel aux trois métiers suivants : loueur de matériel (grue, coffrage, BTP roulant), prestataire de services (montage et maintenance de matériel) et négociant de matériel pour des entreprises du bâtiment et des travaux publics (service complet de mise à disposition de matériel). La démarche compétence, nommée Gestion Prévisionnelle des Emplois et des Compétences, est introduite en 2003 dans un contexte de vieillissement de la population.

Micro-Contrôle (Detchessahar, Devigne et Geoffroy 2007) appartient à un groupe américain d'envergure internationale de 7000 personnes. Le site étudié évolue dans le secteur de la haute technologie et conçoit des microcontrôleurs destinés à être intégrés dans divers biens de consommations courantes ou de haute technologie. Ce domaine d'activité est soumis à de profondes évolutions et à une très forte concurrence notamment sur les délais d'innovation. Conçue en 2000 par le directeur des ressources humaines du site, la démarche s'adresse aux ingénieurs qui représentent $80 \%$ des 280 salariés. Celle-ci vise à favoriser les évolutions de carrières et à résoudre des problèmes récurrents de management.

Modeluxe (Ghaffari et Kogan 2007) est une petite société du secteur du textile et de l'habillement de 70 salariés qui a choisi une stratégie de montée en gamme afin d'éviter la délocalisation vers des pays à bas coût. Cette stratégie repose sur une réputation de qualité des produits livrés, tout en se spécialisant sur les tissus « flous » comme la mousseline ou la soie. Cette évolution a nécessité un profond changement de cap pour l'entreprise avec notamment 
d'importantes restructurations organisationnelles que relaye la démarche compétence initiée en 2004.

Jusqu'en 2007, Silicone (Journé et Melchior 2008) a été l'un des sites industriels d'un grand groupe français. Depuis, c'est une PME de 257 salariés qui fabrique des semi conducteurs pour un chiffre d' affaires de 40 millions d'Euros. En 2002, la direction a engagé une démarche compétence afin de renforcer la capacité des opérateurs à produire sans défaut des produits de haute technologie dans un process de fabrication toujours plus flexible. Cette démarche est intitulée « Programme de développement et de valorisation des compétences du personnel ».

Fondée en 1865, Toutes-Graines (Bretesché S. et Devigne 2009) est l'une des premières entreprises non cotées américaines, dont les capitaux sont essentiellement détenus par les deux familles fondatrices. Le groupe appartient au secteur de l'industrie de la première transformation et du commerce mondial de produits primaires et compte plus de 150000 personnes. Le site étudié comprend 280 personnes. Depuis le début des années 2000, une démarche compétence conçue par le groupe est déployée sur tous les sites.

Nous avons analysé l'ensemble des monographies afin de dégager les similitudes et les singularités de ces démarches. Ce travail a reposé sur la catégorisation des données pour dégager des méta-catégories (Allard-Poesi 2003) construites à partir d'itération entre les données empiriques et les données théoriques (Glaser et Strauss 1967). Quatre méta-catégories ont été élaborées : la logique de la démarche, l'objet de la règle, les points de fragilités et les modes de régulations. Ce travail a alors permis de distinguer quatre figures distinctes de la démarche compétence présentées par la suite.

\section{Les quatre figures de la compétence : qualification locale, optimisation, diversification, articulation local- global}

A travers les cas d'entreprises ici étudiés, les logiques et l'objet de la règle constituent des éléments distinctifs qui permettent de dessiner les «figures » de la compétence qui révèlent des fragilités aux modes de régulation spécifiques.

\subsection{Une démarche pour qualifier localement les compétences}

Dans la première figure, la démarche compétence s'inscrit dans un objectif de qualification de l'activité. L'enjeu porte sur le renouvellement des qualités attendues des salariés au travail. Deux cas sont emblématiques de cette figure : Modeluxe (Ghaffari et Kogan 2007) et Logemer (Bretesché et Chambrier 2007). La démarche compétence est initiée dans le cadre d'un ajustement à de nouveaux marchés impliquant l'augmentation de la valeur ajoutée du produit ou du service. Ce positionnement se traduit par de nouvelles exigences associées au travail : il s'agit de s'adapter en temps réel au cahier des charges défini par le client. Par exemple, la direction de Logemer (Bretesché et Chambrier 2007), un organisme de logement social, fait évoluer les pratiques professionnelles pour répondre aux évolutions du service proposé au locataire. Les postes font l'objet d'un travail de définition des rôles prescrits autour de trois axes : le cœur de métier, les bonnes pratiques et les activités. Chaque salarié se voit ainsi confier un corpus d'items, son «script» qui correspond à un ensemble de comportements attendus. Ici, les outils de gestion définissent des référentiels qui décrivent de «nouvelles façons de travailler », voire les qualités attendues des salariés. La démarche compétence agit comme une règle de travail qui précise les nouveaux comportements exigés dans l'exercice de l'activité. L'adaptation en continu des équipes à des produits complexes et haut de gamme ou aux exigences clients participe à mettre le salarié en situation de faire la preuve de sa performance dans des contextes aux aléas multiples. Par exemple, le référentiel construit chez 
Logemer indique les « rôles professionnels » attendus et les comportements requis tels, la communication, l'échange, l'autonomie ou l'ajustement mutuel au sein d'une même équipe de travail.

Pour les salariés de ces entreprises, la confrontation et la prise en compte des exigences clients fournissent un intérêt renouvelé pour le travail réalisé. Néanmoins, la compétence est devenue très volatile car dépendante des normes du marché et construite en dehors de cadre négocié. Sa définition présente alors des caractéristiques liées à l'entreprise dans laquelle elle s'exerce et par conséquent, ne peut se transposer d'une entreprise à l'autre. Si le salarié est invité à endosser un nouveau rôle professionnel en lien avec les exigences du marché, il n'obtient pas en retour la garantie de pouvoir monnayer ses compétences à l'extérieur du marché. Ainsi, la démarche compétence précise bien de nouvelles règles de travail mais elle ne constitue pas une nouvelle règle de gestion des parcours professionnels.

Le management intermédiaire est alors un acteur majeur de la régulation. A travers l'organisation de l'activité et la distribution du travail et des produits, il participe à reconnaître les compétences et à ordonner la place de chacun dans l'activité. Il assure alors, la confrontation des salariés à des situations nouvelles et donc le développement de leurs compétences. Cet ajustement, qui reste local, limite alors l'obsolescence trop rapide de certaines compétences. Les salariés cherchent aussi à développer leurs compétences individuelles et les faire reconnaître. L'enjeu consiste à être affecté dans les collectifs responsables des services ou produits à plus forte valeur ajoutée pour ensuite argumenter auprès du management intermédiaire cette montée en compétence.

\subsection{Une démarche pour optimiser les ressources humaines}

Toujours dans une logique d'adaptation, la deuxième figure vise ici l'optimisation de la gestion des ressources humaines et à ce titre se focalise sur les règles de gestion. Cette figure a été observée dans deux monographies : Fenêtre (Henriet et Krohmer 2008) et Silicone (Journé et Melchior 2007). Depuis quelques années déjà, leur secteur d'activité s'est transformé faisant de la qualité du produit et la productivité des exigences de premier ordre. Face à ces évolutions, les directions ont fortement repensé l'organisation du travail. Celle-ci combine désormais une forte industrialisation et une flexibilité qualitative (Everaere 1999, 2012) de sa maind'œuvre. Tous ces changements conduisent à une redéfinition des compétences. Par exemple, chez Fenêtre, le menuisier autonome capable de réaliser des pièces entières se transforme en opérateur spécialisé et polyvalent sur une série de tâches. Ces nouvelles exigences se heurtent alors aux systèmes de gestion des ressources humaines en place. Certes ces systèmes évoluent parfois à la marge en fonction des recrutements ou des licenciements en spécifiant alors les nouveaux critères sur lesquels repose la relation d'emploi. Mais ceci ne fait qu'accroître le sentiment d'iniquité des salariés. Rapidement, il devient évident qu'il faut «mettre en ordre » le système de gestion des ressources humaines. Par exemple, le responsable ressources humaines de Fenêtre propose dès 2001, une nouvelle gestion des ressources humaines basée sur les compétences. Pour accompagner l'industrialisation des ateliers, les différents postes de travail ont été étudiés et décomposés. A chacune des compétences un coefficient a été attribué en fonction de son importance : ce coefficient permet de donner une « valeur » à la compétence requise. En plus de ces critères, l'accent est mis sur certaines compétences en fonction des axes stratégiques de l'entreprise. Ce travail d'identification et de cotation des compétences a permis l'élaboration d'un référentiel comptant une soixantaine de compétence. La compétence est désormais utilisée dans les décisions d'affectation, d'évaluation, de formation, de carrière et de rémunération.

Implantée à l'origine dans les ateliers de production, ces démarches concernent avant tout les opérateurs. Elles s'avèrent fortement instrumentalisées et formalisées et sont un outil parmi d'autres pour orienter les comportements des opérateurs. Le processus d'élaboration est piloté par le responsable ressources humaines du site et n'intègre pratiquement pas les salariés. De ce mode de conception, il en découle une attention portée presqu'exclusivement aux compétences requises (Retour 2005). Il s'agit essentiellement de savoir-faire technique et si certains comportements professionnels sont bien sûr requis, ils traduisent majoritairement 
la capacité d'un individu à respecter les règles et les procédures en vigueur dans l'entreprise. La polyvalence est aussi valorisée. Bien évidemment pour être reconnues, ces compétences doivent être effectivement utilisées par les opérateurs. Au final, les compétences évaluées et donc reconnues sont fortement liées à la situation de travail, se déclinent en prescription et doivent être régulièrement utilisées par les opérateurs.

Cette attention portée aux compétences requises fragilise les dynamiques de ces démarches. Liée à la situation de travail, la compétence des opérateurs devient temporaire. Si l'outil de production est modifié ou si le salarié évolue, la compétence détenue n'est tout simplement plus reconnue. Le décalage est alors flagrant entre le temps d'apprentissage nécessaire au développement de compétences, le temps de capitalisation de ces compétences et le besoin presque constant d'adapter celles-ci à la situation qui elle, évolue. Ces systèmes peinent aussi lorsqu'il s'agit d'outiller l'évolution des salariés. Deux raisons peuvent être avancées : le focal sur l'atelier empêche la création d'un marché interne et l'absence de prise en considération de la compétence détenue rend difficile l'identification de potentiel d'évolution.

Certains managers de proximité atténuent ces fragilités en valorisant les compétences détenues, le plus souvent techniques, par des salariés. Ils suggèrent aussi aux pilotes des démarches des ajustements pour mieux prendre en compte le travail des opérateurs. Il arrive alors que les responsables de ressources humaines modifient l'outil. En contrepoint du caractère temporaire de la compétence, certains salariés refusent le principe de la polyvalence. L'enjeu devient alors de bien évaluer la possibilité d'utiliser cette compétence détenue par la suite. Les besoins de production seront-ils suffisamment importants pour que l'opérateur puisse utiliser sa compétence et ainsi la faire reconnaître ? Si oui, l'opérateur s'engage.

\subsection{Une démarche pour accompagner le développement de l'entreprise}

Les démarches construites dans les cas Construction (Charles-Pauvers et Schieb-Bienfait 2008) et Matériel (Charles-Pauvers et Schieb-Bienfait 2008), alimentent le mouvement de diversification des activités. Lancées en 2000 chez Construction et en 2003 chez Matériel, les démarches sont pensées à l'occasion de réflexions stratégiques sur le développement de compétences internes. Projet d'entreprise et évolution des salariés sont ici liés. Par exemple, la démarche de Construction (Charles-Pauvers et Schieb-Bienfait 2008) vise «la construction d'un équilibre entre l'histoire de l'entreprise et les projets personnels». Construite sur un mode de tâtonnement, la démarche vise le développement des compétences individuelles stratégiques. La démarche privilégie le développement de savoirs clés : le management d'équipe, le rôle de tuteur, la gestion de l'entreprise. Faiblement formalisée en termes d'outils de gestion, elle s'appuie sur des entretiens et des groupes participatifs afin de favoriser l'implication des salariés.

Après une phase d'identification des compétences stratégiques, les démarches se traduisent alors par le développement de compétences individuelles stratégiques tant dans le domaine technique que dans le domaine managérial. Afin d'accompagner et de renforcer la maitrise de ces compétences individuelles, les démarches concernent principalement la mise en place de formation et de mobilités internes. Il s'agit alors de repérer les individus détenant des compétences stratégiques, de leur proposer des formations et/ou des mobilités. Des évolutions de carrière sont ainsi offertes à certains salariés. Chez Construction, quelques évolutions exemplaires sont réalisées. Mais rapidement ces démarches trouvent leurs limites. Si des mobilités ont bien lieu, le cloisonnement de certains métiers ne permet cependant pas la création de réels parcours en interne traduisant ainsi l'absence d'une réflexion globale et construite sur la gestion de carrières. Jusqu'à présent, le rythme de croissance de l'entreprise Construction permet le développement de compétences, mais plusieurs salariés s'interrogent : que se passera-t-il en cas de ralentissement de la croissance ? De même, le développement des compétences par la formation devient d'autant plus difficile lorsque le métier n'est pas reconnu sur le marché du travail et que l'apprentissage se fait « sur le tas » comme c'est le cas par exemple chez Matériel. Les processus ressources humaines restent alors largement inachevés. Plus encore, la distinction de certains individus détenteurs de compétences stratégiques pose 
la question de l'évolution de l'ensemble des salariés. Ces démarches perdurent pourtant grâce à quelques points d'appuis venant principalement de l'extérieur. Ces supports, ce sont tout d'abord les divers consultants qui insufflent à plusieurs reprises des dynamiques particulières. Ce sont aussi et surtout les acteurs des branches professionnelles qui ancrent les pratiques de ces entreprises sur un marché du travail plus large. Enfin, les collectifs métiers qui sont des lieux d'apprentissage et de transmission de compétences, assurent collectivement le développement de compétences individuelles stratégiques.

\subsection{Une démarche pour articuler le global et le local}

Dans la quatrième figure, la démarche compétence vise l'articulation entre le niveau global du groupe et les sites locaux de production. Dans les cas étudiés, Micro-Contrôle (Devigne, Detchessahar et Geoffroy 2007) et Toutes-Graines (Bretesché et Devigne 2009), l'outil s'inscrit dans une stratégie de groupe international. Initiée dans le cadre d'un alignement stratégique sur la politique de groupe, l'outil vise tantôt à créer les conditions de la « haute performance » (cas «Toutes-Graines »), tantôt à raccourcir les délais de production et d'innovation (cas « Micro-Contrôle »). Dans un cas comme dans l'autre, il s'agit de « donner la preuve » du niveau de performance atteint par le site et de démontrer sa capacité à s'inscrire dans une stratégie globale. Par exemple, Toutes-Graines (Bretesché et Devigne 2009) se donne pour objectif de doubler en 8 ans son chiffre d'affaires, ses bénéfices et ses effectifs dans le monde. Ces objectifs se fondent sur la mise en œuvre de valeurs : principe du Leadership, composé d'ambition, d'audace et d'exemplarité. Les 3 piliers suivants sont le fondement des comportements et des prises de positions de chaque collaborateur : Haute Performance, Service Client, Innovation. Chaque salarié, doit contribuer à ces valeurs, où qu'il soit et quelle que soit sa position au sein de l'organisation. La haute performance, instituée comme objectif majeur, doit être attestée tant au niveau des résultats du groupe qu'au niveau des comportements des personnes. L'outil d'évaluation des compétences rend compte de cette haute performance dans le comportement des personnes. Il doit également l'inciter.

Au-delà de la valorisation des sites, l'outil organise des marchés internes afin de permettre à certaines catégories d'optimiser leur parcours professionnel. C'est le cas pour les Traders chez Toutes-Graines qui peuvent faire valoir leurs compétences sur différents sites du groupe. L'établissement de règles d'évaluation des compétences a pour effet de valoriser de façon plus spécifique certains profils. La démarche articule ainsi étroitement la performance individuelle à l'échelle locale et celle du groupe à l'échelle internationale.

Mais le dispositif mis en œuvre permet davantage d'esquisser les contours d'une carrière à l'échelle du groupe que d'organiser des filières de promotion ou de mobilité à l'échelle locale. En effet, en fonction du type d'activité conduite, certains salariés peuvent plus facilement se saisir des opportunités offertes par l'évaluation que d'autres. L'outil accentue à ce titre la différenciation des salariés tout en valorisant les compétences jugées stratégiques pour le groupe.

34 Le responsable ressources humaines du site joue alors un rôle important dans la capacité qu'il a de proposer des parcours aux salariés dont les compétences ne sont pas jugées stratégiques par le groupe. Ce mode de régulation atténue partiellement les effets d'une gestion distinctive des compétences et permet alors de réintroduire un peu de collectif mais elle s'avère dans les deux cas bien impuissante face aux politiques portées par le groupe.

Par ailleurs, les compétences sont en partie évaluées à partir de critères qui demandent aux individus d'aller au-delà du travail quotidien. Or ce dépassement est plus ou moins bien vécu en fonction de la nature du travail. Lorsque l'activité requiert une forte indépendance, l'exercice collectif du travail participe à ménager les tâches en plus, voire à aménager des espaces d'entraide pour faire face aux exigences de performance.

\section{Fragilités et modes de régulation à l'occasion des démarches compétences}

Dans chaque «figure», la démarche compétence agit comme de nouvelles règles introduites dans des situations contingentes. Du point de vue des logiques retenues, l'adaptation des 
ressources humaines aux exigences de marchés hautement concurrentiels ou « haut de gamme » participe à définir de nouvelles façons de travailler ou de gérer les postes de travail. De surcroît, la logique de développement des compétences pour accompagner la stratégie de diversification des activités initie de nouvelles règles de travail ou des dispositifs de distinction des compétences individuelles. Ainsi, les démarches compétences remplissent plusieurs fonctions : elles qualifient le travail en tenant compte du repositionnement marché, elles optimisent la gestion des ressources humaines en rationalisant les postes, elles diversifient les compétences et elles participent à articuler la stratégie globale et la performance locale.

L'analyse des huit cas d'entreprise révèle des démarches compétences incomplètes par rapport au modèle de la compétence (Zarifian, 2001). Ces démarches révèlent des fragilités qui sont autant d'occasion pour les acteurs de réguler et d'adapter ces nouvelles exigences (cf. tableau 1).

Tableau 1 : les figures de la démarche compétence entre fragilités et modes de régulation

\begin{tabular}{|c|c|c|c|c|}
\hline & $\begin{array}{l}\text { Figure 1: } \\
\text { qualification } \\
\text { locale }\end{array}$ & $\begin{array}{l}\quad \text { Figure 2: } \\
\text { l'optimisation } \\
\text { des ressources } \\
\text { humaines }\end{array}$ & $\begin{array}{l}\quad \text { Figure 3: le } \\
\text { déploiement du } \\
\text { projet } \\
\text { stratégique }\end{array}$ & $\begin{array}{l}\quad \text { Figure } 4: \\
\text { l'articulation } \\
\text { global/local }\end{array}$ \\
\hline $\begin{array}{l}\text { Logiq } \\
\text { ues }\end{array}$ & Adaptation & Adaptation & $\begin{array}{l}\text { Développem } \\
\text { ent }\end{array}$ & $\begin{array}{l}\text { Développem } \\
\text { ent }\end{array}$ \\
\hline $\begin{array}{l}\text { Règle } \\
\text { s }\end{array}$ & $\begin{array}{l}\text { Règle de } \\
\text { travail }\end{array}$ & $\begin{array}{l}\text { Règle de } \\
\text { gestion }\end{array}$ & $\begin{array}{l}\text { Règle de } \\
\text { travail }\end{array}$ & $\begin{array}{l}\text { Règle } \mathrm{de} \\
\text { gestion }\end{array}$ \\
\hline $\begin{array}{l}\text { Fragil } \\
\text { ités }\end{array}$ & $\begin{array}{l}\text { Compétence } \\
\text { temporaire } \\
\text { Peu de règle } \\
\text { de gestion } \\
\text { Compétence } \\
\text { anonyme }\end{array}$ & $\begin{array}{l}\text { Compétence } \\
\text { temporaire } \\
\text { Peu de règle } \\
\text { de travail } \\
\text { Compétence } \\
\text { anonyme }\end{array}$ & $\begin{array}{l}\text { Peu de règle } \\
\text { de gestion } \\
\text { Compétence } \\
\text { distinctive }\end{array}$ & $\begin{array}{l}\text { Compétence } \\
\text { illimitée } \\
\text { Peu de règle } \\
\text { de travail } \\
\text { Compétence } \\
\text { distinctive }\end{array}$ \\
\hline $\begin{array}{l}\text { Mode } \\
\text { s } \quad \text { de } \\
\text { régulation }\end{array}$ & $\begin{array}{l}\quad \text { Le } \\
\text { management } \\
\text { intermédiaire } \\
\text { réorganise } \\
\text { l'activité } \\
\quad \text { Les salariés } \\
\text { argumentent sur } \\
\text { la «valeur» de } \\
\text { leurs } \\
\text { compétences } \\
\text { individuelles }\end{array}$ & \begin{tabular}{l}
\multicolumn{1}{c}{ Le } \\
management \\
intermédiaire et \\
parfois le \\
responsable \\
ressources \\
humaines \\
reconnaissent la \\
dimension \\
travail Lariés \\
$\quad$ Les salaries \\
investissent leur \\
poste pour \\
développer leurs \\
compétences \\
individuelles
\end{tabular} & $\begin{array}{l}\quad \text { Les } \\
\text { branches } \\
\text { professionnelles } \\
\text { construisent des } \\
\text { règles de } \\
\text { gestion } \\
\quad \text { Les salariés } \\
\text { développent } \\
\text { collectivement } \\
\text { des } \\
\text { compétences } \\
\text { individuelles }\end{array}$ & $\begin{array}{l}\quad \text { Le } \\
\text { responsable } \\
\text { ressources } \\
\text { humaines du } \\
\text { site ajuste à la } \\
\text { marge les règles } \\
\text { aux contraintes } \\
\text { locales } \\
\quad \text { Les salariés } \\
\text { s'appuient sur le } \\
\text { collectif pour se } \\
\text { distinguer }\end{array}$ \\
\hline
\end{tabular}

\subsection{Des compétences fragiles}

L'analyse des différentes figures révèle l'existence de fragilités ne se limitant pas uniquement à une opposition au modèle de la qualification. Deux figures - la qualification locale et l'optimisation des ressources humaines - révèlent des compétences temporaires, très dépendantes des demandes des clients ou des outils de production. En cas de modification de l'un de ces éléments, les salariés se doivent d'oublier les anciennes façons de faire et d'en apprendre des nouvelles. Si ces compétences sont mortelles (Reynaud 2001), c'est notamment parce que le système ne permettant pas de prendre en considération les compétences détenues (Retour 2005) des salariés, il n'est alors pas possible d'opérer une capitalisation des compétences. A l'opposé, dans la figure 4 (articulation globale-locale), les compétences se déclinent à l'infini. Il est ainsi demandé au salarié de démontrer régulièrement qu'il maitrise une nouvelle compétence en plus du stock de compétences déjà existantes. Ces premières 
fragilités révèlent une tension sur le temps de la compétence. D'un côté, les systèmes peinent à se projeter dans l'avenir, ce qui d'ailleurs semble particulièrement problématique avec la mise en place de gestion prévisionnelle des emplois et des compétences. D'un autre côté, les systèmes parce qu'ils conçoivent la compétence comme illimitée, ne permettent pas au salarié d'envisager la progression continue de son apprentissage et donc son évolution au sein de l'organisation.

Ensuite, les démarches compétences parce qu'elles se traduisent par une attention portée plutôt sur le travail ou plutôt sur l'emploi se révèlent largement incomplètes. Dans les figures 2 et 4 (optimisation des compétences et l'articulation globale/locale), la dimension travail est largement absente. Des contradictions peuvent alors voir le jour entre les exigences de gestion des ressources humaines et celles de l'activité au quotidien. Inversement, la qualification locale ou le déploiement du projet stratégique en centrant l'analyse sur le travail ne permettent pas au salarié de faire le lien entre son activité et son parcours au sein de l'organisation.

Enfin, si toutes les démarches compétences ici analysées accompagnent le mouvement d'individualisation des ressources humaines, la distinction individuelle n'est pourtant réellement présente que dans les figures 3 et 4 (déploiement du projet stratégique et articulation global/local). L'outil mise ici sur la distinction des compétences individuelles dites stratégiques. Tout est alors organisé pour identifier, reconnaître et valoriser les salariés détenteurs de ces compétences stratégiques. Se pose alors la question de la gestion des compétences des autres salariés. Dans les figures 1 et 2 (qualification collective et optimisation des ressources humaines), c'est au contraire le caractère anonyme de la compétence qui pose problème. Il s'agit alors pour les salariés de s'adapter et d'acquérir les nouvelles compétences exigées par l'organisation qu'elles que soient les compétences individuelles détenues.

\subsection{Des modes de régulations partiels}

Si les figures de la compétence apparaissent largement incomplètes par rapport au modèle de la compétence, les démarches initiées sont finalement suffisamment malléables pour se révéler relativement robustes. Comme le souligne Denis Segrestin, « Il se pourrait même que cette innovation managériale [la démarche compétence] trouve dans les entraves qui l'encombrent la sagesse que lui refusent ses détracteurs » (Segrestin 2004). C'est précisément cette incomplétude qui d'une certaine façon les rend opératoires et « plastiques » à de multiples usages (Oiry 2006). Les acteurs s'engouffrent dans les incomplétudes du modèle pour élaborer des modes de régulation. Ces régulations portent sur trois objets : le temps, la nature de la règle et la dimension individuelle ou collective.

Quand les démarches compétences envisagent la compétence comme temporaire (figures 1 et 2), les acteurs recherchent la stabilité. Les salariés tentent alors de rester affectés de façon durable dans un poste (figure 2 - l'optimisation des ressources humaines). De même le management de proximité joue sur les affectations des salariés afin de stabiliser les collectifs (figure 1 - la qualification locale). Quand la compétence est conçue comme illimitée, le collectif s'organise pour permettre l'acquisition individuelle de la compétence.

Lorsque les démarches se centrent plus spécifiquement sur la dimension travail, des modes de régulations sont alors développés pour réintroduire la dimension emploi. Ces modes de régulation sont portés par des acteurs en interne comme le management intermédiaire ou de façon plus occasionnelle par des acteurs externes (branches professionnelles mais aussi consultant). A l'inverse, si la règle s'attache à préciser les règles de gestion, le management intermédiaire tente de faire le lien entre ces règles de gestion et le travail au quotidien. Le management de proximité est alors celui qui fait le lien entre travail et emploi : il met en cohérence, ajuste et régule au quotidien.

Lorsque les démarches s'avèrent corrélées aux attentes du marché et dépendantes des modes d'organisation collectives du travail, les salariés mettent en œuvre des stratégies pour se réapproprier à titre individuel leur poste de travail ou voir reconnaître leur « valeur » personnelle. La dimension a priori collective de la démarche compétence induit des stratégies individuelles pour se voir affecter un poste ou un signe tangible de reconnaissance. Lorsque les démarches sont initiées dans le cadre de cycles de développement d'activités et distinguent 
les compétences individuelles, c'est alors le collectif qui « compense » cette individualisation du travail ou de la relation d'emploi. Le groupe permet alors de partager la charge de travail ou le développement de compétences individuelles.

Les salariés et le management de proximité sont des acteurs majeurs de la régulation. Comme le souligne Ughetto (2007, p. 102), « pour ne pas rester paralysés, ils [les salariés et les cadres opérationnels] achèvent le travail que ne pouvait de toute façon pas achever la programmation de la nouvelle activité : identifier les règles qui vont, dans le concret, aider à décider finement des frontières des interventions des uns et des autres, des manières les plus efficaces de se transmettre les dossiers sans perdre de l'information, de se coordonner, et ainsi de suite ». Ce travail de régulation, en réalisant des arbitrages au quotidien, permet aux systèmes de tenir. Des acteurs externes jouent un rôle particulièrement intéressant dans les régulations. C'est le cas par exemple des branches professionnelles qui assurent le développement de parcours reconnus dans et surtout hors de l'entreprise. Ce peut être aussi le cas dans des expériences de gestion territorialisée des emplois et des compétences (Retour et al. 2009). Ici la régulation est déplacée à un niveau inter-organisationnel, niveau qui peut être pertinent pour assurer la transférabilité des compétences.

Les régulations portées par ces différents acteurs révèlent aussi un absent : le responsable ressources humaines. Dans la majeure partie de nos cas, les responsables ressources humaines sont très peu présents dans les processus de régulation. Certains cherchent à ajuster l'outil gestionnaire afin qu'il rende mieux compte des réalités du travail (figure 2 - l'optimisation des ressources humaines) ou encore tentent de maîtriser un dispositif dont l'évolution et la conception leur échappent en partie (figure 4 - l'articulation global/local). Mais ces actions limitées posent directement la question du pilotage des démarches compétences. Certes des régulations ont bien lieu malgré l'absence de pilote. Cependant, si ces modes de régulations permettent de limiter les fragilités des démarches compétences, ils restent néanmoins très localisés et dépendants des acteurs en présence. La transformation des règles à proprement parler se révèle très rare.

\section{Conclusion}

L'analyse des pratiques des organisations révèle des démarches compétences aux figures multiples qui dessinent autant de nouvelles façons de travailler, de produire et de gérer les Hommes. Néanmoins, mises à l'épreuve des faits et des aléas de la production, ces compétences largement incomplètes s'avèrent aussi fragiles. Ces fragilités sont alors l'occasion pour les acteurs de s'emparer ou non de ces démarches en développant des modes de régulation. Les modes de régulation mises en évidence pointent l'importance des régulations autonomes portés par les salariés et celles développées par la hiérarchie intermédiaire. Le management devient alors un acteur central plus ou moins capable de réaliser des compromis entre logique gestionnaire et logique productive. Pourtant ces régulations parce qu'elles ne permettent que très rarement la transformation des règles, restent très locales et en définitive éminemment fragiles. Il convient alors de s'interroger sur le rôle joué par les pilotes des démarches qui dans notre étude se révèlent plutôt absents. Ceci appelle à poursuivre ces études en portant l'attention sur la transformation des règles.

\section{Bibliographie}

Aggeri F. et Labatut J. (2010), « La gestion au prisme de ses instruments. Une analyse généalogique des approches théoriques fondées sur les instruments de gestion », Finance Contrôle Stratégie, vol. 13, $\mathrm{n}^{\circ} 3$, pp. 5-37

Allard-Poesi F. (2003), « Coder les données », dans Y. Giordano (coord.), Conduire un projet de recherche. Une perspective qualitative. EMS. Éd. Management \& société, pp. 246-290

Autès M. (2004), Les paradoxes du travail social, 2ème Ed., Lavoisier.

Bouteiller D. (1997), « Le syndrome du crocodile et le défi de l'apprentissage continu », Revue Internationale de Gestion, vol. 22, $\mathrm{n}^{\circ} 3$, p. 14-25 
Courpasson D. et Livian Y-F (1991), « Le développement récent de la notion de « compétence »: glissement sémantique ou idéologie ? », Revue de Gestion des Ressources Humaines, octobre, p. 3-9

Defélix C., Retour D. et Dietrich A. (2007), « Outils et modèles de la gestion des compétences en France : bilan et défis pour les années 2000 », XVIII Congrès de l'AGRH, Fribourg, septembre

Detchessahar M. et Journé B. (2007), « Une approche narrative des outils de gestion », Revue Française de Gestion, $n^{\circ} 174$, pp. 77-92

Dietrich A. (2002), «Les paradoxes de la notion de compétence en gestion des ressources humaines », Revue Sciences de Gestion, ${ }^{\circ}$ 33, pp. 97-121

Eustache D. (2001), « Politique salariale, régulation et échange social », Revue Française de Sociologie, vol. 42, $\mathrm{n}^{\circ} 2$, pp. 295-326

Everaere C. (2012), «Flexibilité et ressources humaines : compatibilités et contradictions », Revue Française de Gestion, $n^{\circ} 221$.

Everaere C. (2000), « La compétence : un compromis multidimensionnel fragile », Gestion 2000, juilletaoût

Everaere, C. (1999), «Emploi, travail et efficacité de l'entreprise : les effets pervers de la flexibilité quantitative », Revue Française de Gestion, juin-juillet-août

Gilbert P. (2003), « Jalons pour une histoire de la gestion des compétences », dans A. Klarsfeld et E. Oiry (coord.), Gérer les compétences. Des instruments aux processus, Vuibert, pp. 11-32

Gilbert P. et Mader C. (1999), «L'appréciation des compétences est-elle rentable », dans P. Gilbert et G. Schmidt (dir.), Evaluation des compétences et situations de gestion, Economica, pp. 15-30

Glaser B. And Strauss A. (1967), The discovery of Grounded Theory : Strategies for Qualitative Research. Aldine

Havard C. et Krohmer C. (2008), «Création et articulation des règles dans le cadre d'un management des compétences ». Revue de Gestion des Ressources Humaines, octobre-novembre-décembre

Jouvenot C et Parlier M (2005), Élaborer des référentiels de compétences, Éditions de ANACT

Joyeau A. et Retour D. (1999) : «La gestion prévisionnelle des emplois et des compétences entre contrôle et autonomie », Revue de gestion de ressources humaines, juillet-août

Monchatre S. (2004), « De l'ouvrier à l'opérateur : chronique d'une conversion », Revue Française de Sociologie, vol. $45, \mathrm{n}^{\circ} 1$

Monchatre S. (2002), «Les avatars du modèle de la compétence. L'exemple d'un site de la sidérurgie », Formation Emploi, $\mathrm{n}^{\circ} 77$, pp. 51-68

Oiry E. (2006), « La dynamique des instrumentations de gestion par les compétences - Proposition d'un cadre d'analyse », dans C. Defélix, A. Klarsfeld, E. Oiry, Nouveaux regards sur la gestion des compétences, Vuibert, pp. 13-31

Oiry E. et Sulzer F. (2002), « Le référentiels de compétences, enjeux et formes », dans D. Brochier (dir.), La gestion des compétences. Acteurs et pratiques, Economica, pp. 29-47

Oiry E. et D'Iribarne A. (2001), « La notion de compétence : continuité et changements par rapport à la notion de qualification », Sociologie du travail, vol. $43, \mathrm{n}^{\circ} 1$, pp. 49-66

Paradeise C. et Lichtenberger Y. (2001), « Compétence, compétences », Sociologie du travail, vol. 43, $\mathrm{n}^{\circ} 1$, pp. 33-48

Parlier M. (2011), « Gérer les compétences en PME », dans Louart P. et Vilette M-A (coord.), La GRH dans les PME, Vuibert, pp. 175-198

Pichault F. (dir.) (2006), Faut-il brûler la gestion des compétences ? Une exploration des pratiques en entreprise, De Boeck \& Larcier

Retour D., Picq T. et Defélix C. (2009), Gestion des compétences. Nouvelles relations, nouvelles dimensions, Vuibert

Retour D. (2005), « Le DRH de demain face au dossier compétence », Management et Avenir, $n^{\circ} 4$

Retour D. et Rapiau M.-T. (2006), « Gestion des compétences et formation au sein de l'entreprise », Cahiers Français, $n^{\circ} 333$, pp. $76-81$

Reynaud J.D. (2001), «Le management par les compétences. Un essai d'analyse », Sociologie du travail, vol. $45, \mathrm{n}^{\circ} 1$

Reynaud J.-D. (1997), Les règles du jeu - L'action collective et la régulation sociale, Armand Colin 
Reynaud J.-D. (1988), «Les régulations dans les organisations », Revue Française de Sociologie, vol. 29, $\mathrm{n}^{\circ} 1$

Richebé N. (2002), «Les réactions des salariés à la "logique compétence" : vers un renouveau de l'échange salarial ?», Revue Française de Sociologie, vol. 43, n 2, pp. 99-126

Segrestin D. (2004), Les chantiers du manager. Armand Colin

Ughetto P. (2007), Faire face aux exigences du travail contemporain. Conditions de travail et management, Éditions de l'ANACT

Zarifian P. (2009), Le travail et la compétence : entre puissance et contrôle, Le travail humain, PUF

Zarifian P. (2001), Le modèle de la compétence. Editions Liaisons

Zarifian P. (1988), «L'émergence du modèle de la compétence », dans F. Stankiewicz (dir), Les stratégies d'entreprises face aux Ressources Humaines. L'après Taylorisme. Economica

Les monographies :

Bretesché S. et Chambrier L. (2007), Logemer

Bretesché S. et Devigne M. (2009), Toutes-Graines

Charles-Pauvers B. et Schieb-Bienfait N. (2008), Matériel

Charles-Pauvers B. et Schieb-Bienfait N. (2008), Construction

Detchessahar M., Devigne M et Geoffroy B. (2007), Micro-Contrôle

Ghaffari S. et Kogan A.-F (2007), Modeluxe

Henriet B. et Krohmer C. (2008), Fenêtre

Journé B. et Melchior E. (2008), Silicone

Ces monographies sont reprises pour partie dans l'ouvrage Fragiles compétences coordonné par Bretesché S. et Krohmer C. (2010), Presses des Mines

\section{Pour citer cet article}

Référence électronique

Cathy Krohmer et Sophie Bretesché, «Fragilités et modes de régulation des figures de la compétence », Finance Contrôle Stratégie [En ligne], 16-2 | 2013, mis en ligne le 12 juillet 2013, consulté le 10 décembre 2014. URL : http://fcs.revues.org/1362

\section{À propos des auteurs}

\section{Cathy Krohmer}

Chercheur au Laboratoire d'Economie et de Sociologie du Travail (LEST) de l'Université d'Aixmarseille.

\section{Sophie Bretesché}

Chercheur au Laboratoire d'Economie et de Management Nantes-Atlantique (LEMNA) de l'Université de Nantes.

\section{Droits d'auteur}

Tous droits réservés

\section{Résumés}

Cet article propose une analyse des démarches compétences portant à la fois sur leurs logiques et sur la nature des règles qu'elles initient dans les organisations. La recherche s'est appuyée sur l'analyse de données secondaires issues de huit monographies. Ces monographies présentent les démarches compétences d'entreprises de secteurs variés. Quatre figures de la compétence se distinguent en termes d'objet de la règle et de logique. De nature hybride, les démarches présentent des incomplétudes qui dessinent des modes de régulation partiels. 
This article proposes an analysis of competency-based management related to its rationale and the nature of the rules which it initiates in organizations. This research is based on the study of eight cases of companies from various sectors whose competency-based management practices have been categorized. Four competency "figures" are distinguished. Competencybased management practices present incompleteness represent partial modes of regulation.

\section{Entrées d'index}

Mots-clés : démarche compétence, règles, régulations

Keywords : competency-based management, rules, strategic context, regulation

Code JEL : J24 - Human Capital; Skills; Occupational Choice; Labor Productivity 